Supplement of Hydrol. Earth Syst. Sci., 22, 3473-3491, 2018

https://doi.org/10.5194/hess-22-3473-2018-supplement

(C) Author(s) 2018. This work is distributed under

the Creative Commons Attribution 4.0 License.

(c) (1)

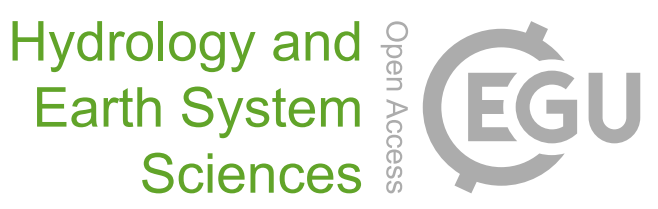

Supplement of

\title{
Delineating multiple salinization processes in a coastal plain aquifer, north- ern China: hydrochemical and isotopic evidence
}

\author{
Dongmei Han and Matthew J. Currell \\ Correspondence to: Dongmei Han (handm@igsnrr.ac.cn)
}

The copyright of individual parts of the supplement might differ from the CC BY 4.0 License. 

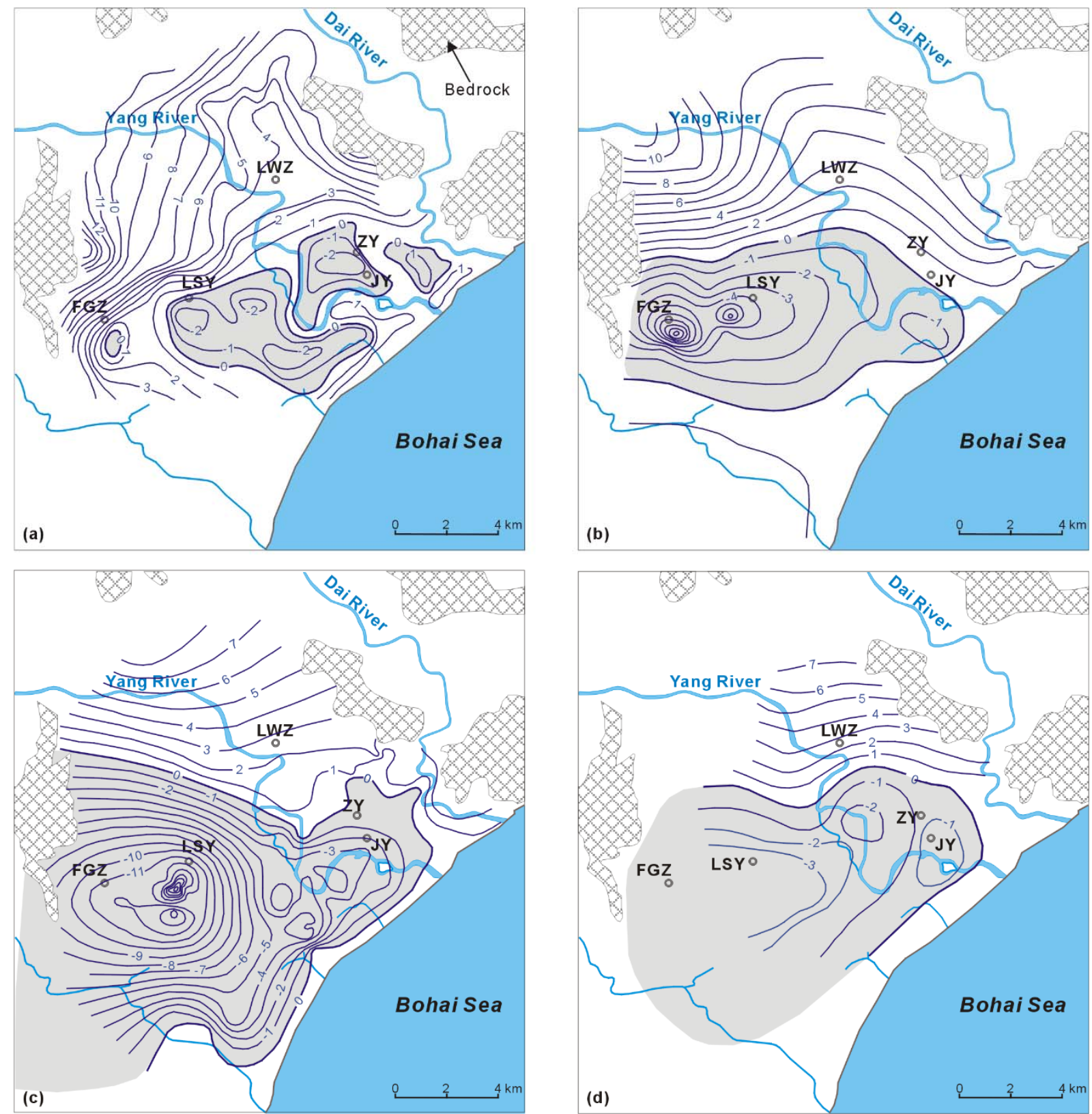

Fig. S1 Maps showing the distribution of water table contours in shallow aquifer (a) in 1986 (from Han, 1988), (b) in 1998 (from Zuo, 2006), (c) in 2004 (from Zuo, 2006), and (d) in 2010 (this study). The depression area (gray zone) refers to the potential area enclosed by 0 m.a.s.l. contour line of water tables. 


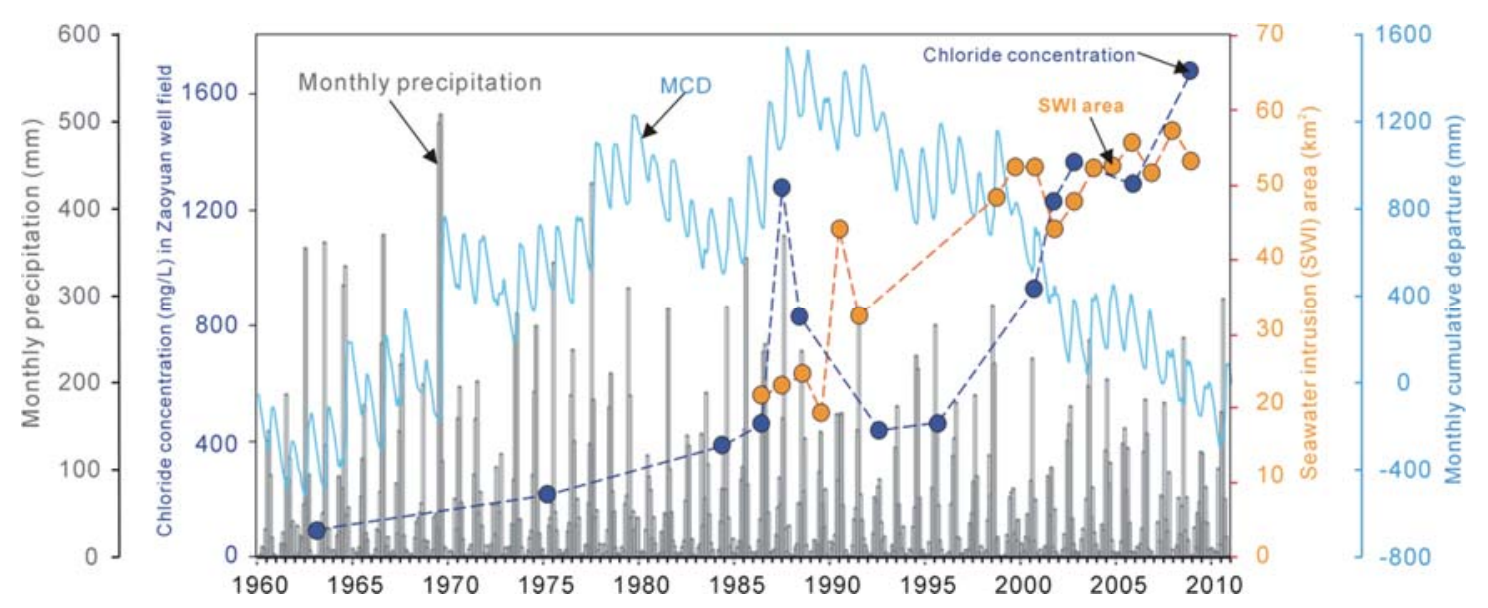

Fig. S2 Graph showing the temporal variation of monthly cumulative rainfall departure (CRD, Weber and Stewart, 2004), monthly precipitation, average concentration of chloride in groundwater (dark blue) and surface area with $>250 \mathrm{mg} \mathrm{Cl} / \mathrm{L}$ (yellow) between 1963 and 2008 (data from Zang et al., 2010).

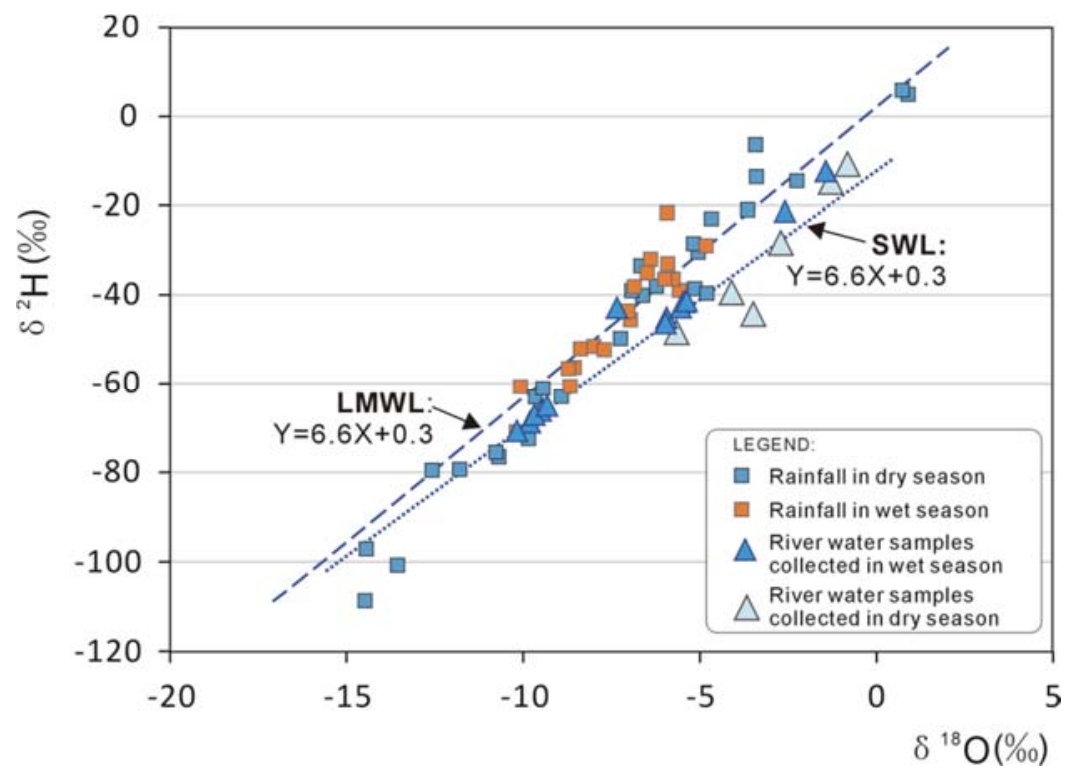

Fig. S3 Graph showing $\delta^{2} \mathrm{H}$ vs. $\delta^{18} \mathrm{O}$ of water samples in rainfall and river water. Dry season- July to October; wet season- November to June. 
Table $\mathrm{S} 1 . \mathrm{NO}_{3} / \mathrm{Cl}$ and $\mathrm{Sr} / \mathrm{Cl}$ ratios in water samples

\begin{tabular}{|c|c|c|c|c|c|c|c|}
\hline WaterType & ID & $\begin{array}{c}\text { Sampling } \\
\text { Time }\end{array}$ & $\begin{array}{c}\mathrm{Cl} \\
\mathrm{mg} / \mathrm{L}\end{array}$ & $\begin{array}{l}\mathrm{NO}_{3} \\
\mathrm{mg} / \mathrm{L}\end{array}$ & $\begin{array}{c}\mathrm{Sr} \\
\mathrm{mg} / \mathrm{L}\end{array}$ & $\mathrm{NO}_{3} / \mathrm{Cl}$ & $\begin{array}{r}\mathrm{Sr} / \mathrm{Cl} \\
\left(\times 10^{-3}\right)\end{array}$ \\
\hline \multicolumn{8}{|c|}{ Shallow groundwater samples: } \\
\hline \multirow{25}{*}{ 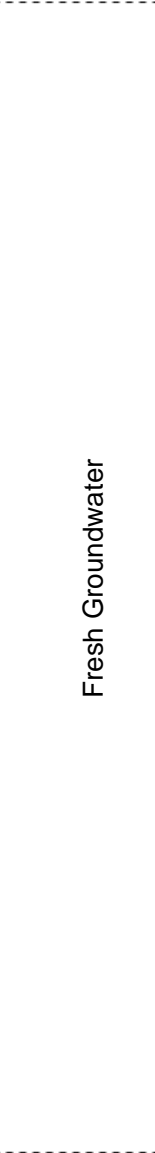 } & G4 & Aug.2010 & 124.3 & 92.6 & 0.67 & 0.745 & 5.41 \\
\hline & G27 & Aug.2010 & 177.5 & 163.0 & 1.22 & 0.918 & 6.88 \\
\hline & G12 & Aug.2010 & 276.9 & 31.9 & 0.25 & 0.115 & 0.90 \\
\hline & G17 & Aug.2010 & 88.8 & 39.5 & 0.33 & 0.445 & 3.68 \\
\hline & G18 & Aug.2010 & 124.3 & 137.8 & 0.71 & 1.109 & 5.71 \\
\hline & G1 & Sep.2009 & 220.1 & 2.0 & 0.66 & 0.009 & 3.00 \\
\hline & G4 & Sep.2009 & 124.3 & 178.5 & 0.85 & 1.437 & 6.83 \\
\hline & G5 & Sep.2009 & 303.4 & 162.3 & 1.14 & 0.535 & 3.74 \\
\hline & G23 & Sep.2009 & 88.8 & 163.4 & 0.55 & 1.841 & 6.17 \\
\hline & G7 & Sep.2009 & 81.7 & 41.2 & 0.41 & 0.505 & 5.01 \\
\hline & G8 & Sep.2009 & 334.6 & 85.4 & 1.19 & 0.255 & 3.57 \\
\hline & G11 & Sep.2009 & 222.9 & 74.5 & 0.84 & 0.334 & 3.75 \\
\hline & G12 & Sep.2009 & 174.0 & 46.0 & 0.53 & 0.264 & 3.06 \\
\hline & G28 & Sep.2009 & 127.8 & 22.5 & 0.58 & 0.176 & 4.54 \\
\hline & G18 & Sep.2009 & 110.1 & 152.9 & 0.65 & 1.389 & 5.94 \\
\hline & G20 & Sep.2009 & 246.9 & 107.1 & 3.94 & 0.434 & 15.94 \\
\hline & G17 & Sep.2009 & 243.5 & 126.4 & 0.71 & 0.519 & 2.91 \\
\hline & G3 & Jun.2008 & 315.0 & & 0.46 & & 1.47 \\
\hline & G4 & Jun.2008 & 74.6 & 75.8 & 0.47 & 1.017 & 6.29 \\
\hline & G5 & Jun.2008 & 310.9 & 119.5 & 1.43 & 0.384 & 4.61 \\
\hline & G8 & Jun.2008 & 349.7 & 55.9 & 1.08 & 0.160 & 3.07 \\
\hline & G12 & Jun.2008 & 281.0 & 29.8 & 0.19 & 0.106 & 0.68 \\
\hline & G17 & Jun.2008 & 227.9 & 92.6 & 0.57 & 0.406 & 2.50 \\
\hline & G18 & Jun.2008 & 115.5 & 144.2 & 0.41 & 1.249 & 3.55 \\
\hline & G20 & Jun.2008 & 234.3 & 18.2 & 2.10 & 0.078 & 8.95 \\
\hline \multirow{20}{*}{ 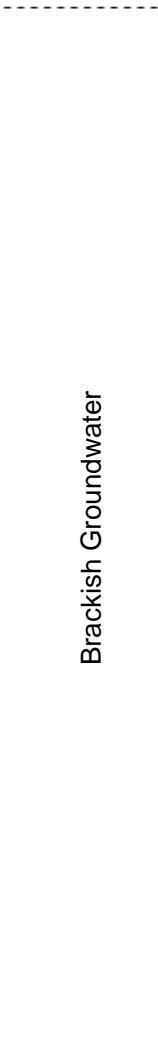 } & $\mathrm{G} 1$ & Aug.2010 & 312.4 & 120.0 & 0.54 & 0.384 & 1.71 \\
\hline & G3 & Aug.2010 & 654.3 & 106.0 & 0.97 & 0.162 & 1.49 \\
\hline & G15 & Aug.2010 & 435.7 & 181.3 & 1.60 & 0.416 & 3.67 \\
\hline & G26 & Aug.2010 & 784.6 & 339.4 & 1.20 & 0.433 & 1.53 \\
\hline & G11 & Aug.2010 & 646.1 & 414.1 & 1.51 & 0.641 & 2.34 \\
\hline & G20 & Aug.2010 & 596.4 & 177.9 & 3.95 & 0.298 & 6.62 \\
\hline & G5 & Aug.2010 & 447.3 & 253.3 & 0.78 & 0.566 & 1.74 \\
\hline & G8 & Aug.2010 & 596.4 & 141.4 & 1.87 & 0.237 & 3.13 \\
\hline & G7 & Aug.2010 & 299.6 & 338.3 & 0.66 & 1.129 & 2.20 \\
\hline & G19 & Aug.2010 & 600.0 & 211.9 & 7.10 & 0.353 & 11.84 \\
\hline & $\mathrm{G} 24$ & Aug.2010 & 646.1 & 952.1 & 2.44 & 1.474 & 3.78 \\
\hline & $\mathrm{G} 22$ & Sep.2009 & 408.3 & 2.0 & 0.70 & 0.005 & 1.72 \\
\hline & G10 & Sep.2009 & 2563.1 & 27.7 & 1.92 & 0.011 & 0.75 \\
\hline & G14 & Sep.2009 & 291.1 & 109.8 & 0.82 & 0.377 & 2.81 \\
\hline & G24 & Sep.2009 & 454.4 & 441.5 & 0.93 & 0.972 & 2.05 \\
\hline & G19 & Sep.2009 & 622.0 & 91.5 & 4.87 & 0.147 & 7.83 \\
\hline & G1 & Jun.2008 & 717.1 & 87.7 & 0.17 & 0.122 & 0.24 \\
\hline & G11 & Jun.2008 & 451.2 & & 1.00 & & 2.21 \\
\hline & G14 & Jun.2008 & 372.8 & 267.3 & 0.93 & 0.717 & 2.48 \\
\hline & G15 & Jun.2008 & 1675.6 & 146.8 & 2.17 & 0.088 & 1.29 \\
\hline
\end{tabular}




\begin{tabular}{|c|c|c|c|c|c|c|c|}
\hline \multicolumn{8}{|c|}{ Deep Groundwater samples: } \\
\hline \multirow{3}{*}{$\begin{array}{c}\text { Fresh } \\
\text { Groundwater }\end{array}$} & G25 & Aug.2010 & 68.1 & 71.0 & 0.25 & 1.042 & 3.66 \\
\hline & G16 & Sep.2009 & 214.4 & & 2.68 & & 12.51 \\
\hline & G29 & Sep.2009 & 255.6 & 26.3 & 0.20 & 0.103 & 0.77 \\
\hline \multirow{10}{*}{ 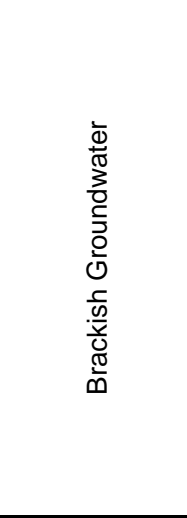 } & G29 & Aug.2010 & 803.4 & 143.0 & 6.95 & 0.178 & 8.65 \\
\hline & G16 & Aug.2010 & 766.8 & 5.5 & 4.00 & 0.007 & 5.21 \\
\hline & G9 & Jun.2008 & 823.6 & 47.4 & 7.40 & 0.058 & 8.98 \\
\hline & G9 & Sep.2009 & 917.4 & 65.8 & 8.02 & 0.072 & 8.74 \\
\hline & G9 & Aug.2010 & 1228.3 & 337.4 & 11.59 & 0.275 & 9.44 \\
\hline & G14' & Aug.2010 & 553.8 & 433.7 & 1.06 & 0.783 & 1.91 \\
\hline & G13 & Aug.2010 & 908.8 & 210.6 & 0.26 & 0.232 & 0.29 \\
\hline & G13 & Jun.2008 & 945.4 & & 0.55 & & 0.58 \\
\hline & G13 & Sep.2009 & 882.0 & & 0.35 & & 0.39 \\
\hline & $\mathrm{G} 2$ & Jun.2008 & 1093.4 & & 1.03 & & 0.94 \\
\hline \multicolumn{8}{|c|}{ River water samples: } \\
\hline \multicolumn{8}{|c|}{ Fresh water samples } \\
\hline Dai River & S9 & Aug.2010 & 80.3 & 65.2 & 0.34 & 0.813 & 4.20 \\
\hline Dai River & $\mathrm{S} 12$ & Aug.2010 & 71.3 & 41.9 & 0.30 & 0.588 & 4.14 \\
\hline Dai River & S8 & Aug.2010 & 68.6 & 54.9 & 0.32 & 0.801 & 4.67 \\
\hline Yang River & S6 & Aug.2010 & 66.0 & 51.9 & 0.30 & 0.786 & 4.53 \\
\hline Yang River & $\mathrm{S} 2$ & Aug.2010 & 63.2 & 40.2 & 0.26 & 0.636 & 4.10 \\
\hline Yang River & S5 & Sep.2009 & 85.2 & 12.9 & 0.36 & 0.152 & 4.27 \\
\hline Yang River & S4 & Sep.2009 & 733.1 & 6.6 & & 0.009 & \\
\hline Yang River & S6 & Sep.2009 & 99.4 & 6.6 & 0.30 & 0.067 & 3.04 \\
\hline Dai River & s9 & Sep.2009 & 88.8 & 6.8 & 0.36 & 0.077 & 4.03 \\
\hline Dai River & S8 & Sep.2009 & 174.0 & 6.4 & 0.55 & 0.037 & 3.17 \\
\hline Yang River & S6 & Jun.2008 & 81.7 & 12.6 & 0.31 & 0.154 & 3.82 \\
\hline Dai River & $\mathrm{S} 10$ & Jun.2008 & 208.9 & 23.1 & 0.57 & 0.111 & 2.72 \\
\hline Dai River & s9 & Jun.2008 & 92.3 & 7.5 & 0.35 & 0.081 & 3.81 \\
\hline \multicolumn{8}{|c|}{ Brackish and salt water samples } \\
\hline Yang River & S3 & Sep.2009 & 11289.4 & & 4.29 & & 0.38 \\
\hline Dai River & $\mathrm{S} 12$ & Sep.2009 & 16766.3 & & 7.64 & & 0.46 \\
\hline Dai River & $\mathrm{S} 11$ & Sep.2009 & 1601.5 & 2.8 & 0.93 & 0.002 & 0.58 \\
\hline Yang River & $\mathrm{S} 1$ & Jun.2008 & 14953.5 & & 5.55 & & 0.37 \\
\hline Yang River & $\mathrm{S} 2$ & Jun.2008 & 8328.3 & & 3.37 & & 0.40 \\
\hline \multirow[t]{2}{*}{ Dai River } & $\mathrm{S} 7$ & Jun.2008 & 16677.1 & & 6.36 & & 0.38 \\
\hline & SW1 & Aug.2010 & 14768.3 & 810.1 & 5.79 & 0.055 & 0.39 \\
\hline \multirow[t]{2}{*}{ Seawater: } & SW1 & Sep.2009 & 16568.0 & & 6.45 & & 0.39 \\
\hline & sw2 & Sep.2009 & 14484.8 & & 5.43 & & 0.37 \\
\hline
\end{tabular}

Note: $\mathrm{NO}_{3} / \mathrm{Cl}$ and $\mathrm{Sr} / \mathrm{Cl}$ - mass ratios 